\title{
Large-angle Rayleigh scattering of linearly polarized, hard synchrotron $x$ rays by krypton and xenon
}

\author{
F. Smend, D. Schaupp, ${ }^{*}$ H. Czerwinski, ${ }^{\dagger}$ and M. Schumacher \\ II. Physikalisches Institut der Universität Göttingen, D-3400 Göttingen, Federal Republic of Germany \\ A. H. Millhouse $\ddagger$ \\ Fachrichtung 11.4, Universität des Saarlandes, D-6600 Saarbrücken 11, Federal Republic of Germany \\ Lynn Kissel \\ Test Planning and Diagnostics Division, Sandia National Laboratories, Albuquerque, New Mexico 87185
}

(Received 8 June 1987)

\begin{abstract}
Using linearly polarized synchrotron $\mathrm{x}$ rays with energies $E=21.2-43.7 \mathrm{keV}$, differential cross sections for large-angle elastic photon scattering by $\mathrm{Kr}$ and $\mathrm{Xe}$ were measured. In the case of $\mathrm{Xe}$, the energy region close to the $K$-shell binding energy $\left(B_{K}=34.561 \mathrm{keV}\right)$ was investigated in detail. The experimental results agree well with theoretical predictions based upon the numerical energyshifted second-order $S$-matrix theory, and with high-energy-limit-corrected anomalous-scatteringfactor predictions.
\end{abstract}

\section{INTRODUCTION}

The investigation of elastic photon scattering by atoms has made considerable progress during the last ten years, with respect to theory as well as to experiment. For the theoretical calculation of Rayleigh scattering amplitudes, the second-order $S$-matrix formalism of quantum electrodynamics, originally formulated for hydrogenlike wave functions, has been adapted to self-consistent Dirac-Hartree-Fock-Slater (DHFS) wave functions of the scattering electrons (see the review papers, Refs. 1-3). During the same time, a considerable amount of experimental data has been obtained using semiconductor photon detectors of good energy resolution (see, e.g., Refs. 4-6 and further references listed in Ref. 3).

At $\mathrm{x}$-ray energies, and $\gamma$-ray energies below $800 \mathrm{keV}$, elastic photon scattering is described as a coherent superposition of Rayleigh $(R)$ scattering by virtual excitation of the bound electrons and of nuclear Thomson $(T)$ scattering by the nucleus as a point charge. There is, in general, very good agreement between experimental results and theoretical predictions. ${ }^{3}$ Above $800 \mathrm{keV}$, Delbrück $(D)$ scattering by the Coulomb field of the nucleus and nuclear Rayleigh $(N)$ scattering by virtual excitation of the giant dipole resonance contribute, at least for heavy nuclei. ${ }^{6,7}$

The experiments listed in Ref. 3 used unpolarized photons having fixed, discrete energies $E$. With the exception of one experiment carried out with characteristic $\mathrm{x}$ rays, ${ }^{8}$ the photons were provided by radioactive sources. The selection of photon energies was governed by technical rather than physical criteria (availability, half-life and $\gamma$ spectra of radioactive nuclides, etc.). As a consequence, a systematic investigation of the "anomalous" energy dependence of the scattering cross section at photon energies near the $K$ absorption edge of the scattering atoms could not be performed.

During the last few years large electron storage rings have become powerful sources of photons in the hard-xray regime. The synchrotron radiation (SR) emitted by the orbiting electrons has a continuous spectrum, from which a monochromator may select quasimonochromatic photons having a continuously variable energy $E$. In addition, the photon beam has a strong (but not complete) linear polarization.

The aim of the present work was to use for the first time, SR for the systematic investigation of Rayleigh scattering at photon energies $E$ above $20 \mathrm{keV}$ and large scattering angles $\Theta$, where Compton scattering is well resolved from elastic scattering by present-day semiconductor detectors. The monatomic gases $\mathrm{Kr}$ and $\mathrm{Xe}$ were selected as scattering targets, thus eliminating possible molecular or solid-state effects on the scattering process.

\section{THEORY}

Elastic scattering of a photon by an atom is a process in which no energy is transferred to the internal degrees of freedom of the atom and no additional photons are radiated. In our description of elastic scattering, we assume that the scattering occurs as an isolated photonatom event. We also assume that no internal degrees of freedom of the atom are observed, and that, in particular, the target atoms are randomly oriented.

With these assumptions, the observables of the elastic scattering process are the momentum and polarization of the incident photon $\left(\mathbf{k}_{i}, \epsilon_{i}\right)$ and the scattered photon $\left(\mathbf{k}_{f}, \boldsymbol{\epsilon}_{f}\right)$. Scattering is discussed in terms of a differential scattering amplitude $A$ that is related to the differential scattering cross section by

$$
d \sigma / d \Omega=|A|^{2} .
$$


With this definition, the normalization of $A$ is defined except for a phase.

The general form of the scattering amplitude is

$$
\boldsymbol{A}=\boldsymbol{\epsilon}_{i} \cdot \boldsymbol{\epsilon}_{f}^{*} F\left(\mathbf{k}_{i} \cdot \mathbf{k}_{f}\right)+\boldsymbol{\epsilon}_{i} \cdot \hat{k}_{f} \boldsymbol{\epsilon}_{f}^{*} \cdot \hat{k}_{i} \boldsymbol{G}\left(\mathbf{k}_{i} \cdot \mathbf{k}_{f}\right),
$$

where $\hat{k}_{i}=\mathbf{k}_{i} / k_{i}, \quad k_{i}=\left|\mathbf{k}_{i}\right|, \quad \hat{k}_{f}=\mathbf{k}_{f} / k_{f}, \quad$ and $k_{f}$ $=\left|\mathbf{k}_{f}\right|$. For elastic scattering, $k_{i}=k_{f}=k=\omega / c$. The rotationally invariant amplitudes $F$ and $G$ are complex and depend upon the photon energy $E=\hbar \omega$ and atomic properties such as the nuclear charge $Z$, but only depend on the vectors of the problem through the inner product $\mathbf{k}_{i} \cdot \mathbf{k}_{f}$. This inner product may be written in terms of $\hbar q$, the magnitude of the momentum transferred to the atom, $\hbar \mathbf{k}_{i}-\hbar \mathbf{k}_{f}$, as

$$
\begin{aligned}
q^{2}=k_{i}^{2}+k_{f}^{2}-2 \mathbf{k}_{i} \cdot \mathbf{k}_{f} & =2 k^{2}(1-\cos \Theta) \\
& =4 k^{2} \sin ^{2}(\Theta / 2),
\end{aligned}
$$

where $\Theta$ is the scattering angle between $\hat{k}_{i}$ and $\hat{k}_{f}$.

In addition to the pair of rotationally invariant amplitudes $F$ and $G$, other choices are often made. For linear photon polarization, the photon polarization vectors are resolved into components parallel and perpendicular to the scattering plane (containing the vectors $\mathbf{k}_{i}$ and $\mathbf{k}_{f}$ ) as

$$
\epsilon=\epsilon^{\|} \hat{\boldsymbol{\epsilon}}^{\|}+\epsilon^{\perp} \hat{\epsilon}^{\perp} .
$$

One can then express the scattering amplitude $A$ in terms of linear amplitudes $A_{\|}$and $A_{\perp}$ as

$$
A=\epsilon_{i}^{\|} \epsilon_{f}^{*} A_{\|}+\epsilon_{i}^{\perp} \epsilon_{f}^{\perp *} A_{\perp},
$$

where

$$
\begin{aligned}
& A_{\|}=F \cos \Theta-G \sin ^{2} \Theta, \\
& A_{\perp}=F .
\end{aligned}
$$

In terms of the linear amplitudes, the cross sections for scattering of linearly polarized photons are given as

$$
\begin{aligned}
& d \sigma_{\|} / d \Omega=\left|A_{\|}\right|^{2}, \\
& d \sigma_{\perp} / d \Omega=\left|A_{\perp}\right|^{2} .
\end{aligned}
$$

The atom is a composite system and all its components contribute to the scattering of a photon. To a good approximation for $\mathrm{x}$ rays and low-energy $\gamma$ rays, the amplitude for scattering from an atom can be written as the sum of contributions from the bound electrons (Rayleigh scattering $R$ ), the nucleus (nuclear Thomson scattering $T$ and nuclear Rayleigh scattering $N$ ), the virtual pair creation in the field of the nucleus (Delbrück scattering D) as

$$
A=A^{R}+A^{T}+A^{N}+A^{D} .
$$

For the $\mathrm{x}$-ray energies considered in this paper, the primary contribution to $A$ is from $A^{R}$, with a very much smaller contribution from $A^{T}$, and negligible contributions from $A^{N}$ and $A^{D}$.

Currently, the best predictions for Rayleigh scattering of $\mathrm{x}$ rays and low-energy $\gamma$ rays are based on the $S$ matrix approach (see, for example, Refs. 2 and 3). The starting point is Furry's extension of the FeynmanDyson formulation of quantum electrodynamics, in which the interaction of electrons and positrons with the atomic field is included in the unperturbed Hamiltonian, while the interaction with the radiation field is treated as a perturbation. Rayleigh scattering is calculated as a second-order single-electron transition process from electrons bound in a relativistic self-consistent central potential. Using this approach, the problem reduces to the numerical evaluation of one-dimensional integrals over radial solutions to inhomogeneous Dirac equations. Ideally, one should compute amplitudes for all atomic electrons following this approach. In practice, due to limitations in computer time and accuracy, amplitudes for the outer weakly bound electrons are generally obtained in modified form-factor approximation.

For the photon energies considered in this paper, the contribution due to the nuclear Thomson amplitude is small as compared with the Rayleigh amplitude. We model nuclear Thomson scattering as scattering from a point-charge particle,

$$
\begin{aligned}
& A_{\perp}^{T}=-r_{0} m Z^{2} / M, \\
& A_{\|}^{T}=A_{\perp}^{T} \cos \Theta,
\end{aligned}
$$

where the classical electron radius is $r_{0}=e^{2} / m c^{2}$ $\approx 2.82 \times 10^{-13} \mathrm{~cm}$, and $m / M$ is the ratio of the electron rest mass to that of the nucleus.

Within about $1 \mathrm{keV}$ of atomic thresholds, the $S$-matrix approach for the calculation of Rayleigh amplitudes must be modified. The relativistic single-particle central potential does not predict inner-shell atomic thresholds with an accuracy better than about $1 \%$. To improve the $S$-matrix predictions near thresholds, an energy-scaling procedure has been developed ${ }^{9}$ taking care of the small difference between predicted and experimental electron binding energy. This energy-scaling procedure extends the usefulness of $S$-matrix predictions closer to atomic thresholds.

A simpler approach to the calculation of Rayleigh scattering is to treat the scattering as being due to free electrons having, however, a spatial distribution of their respective probability density as determined by their binding to the atom. If the scattering is from an extended charge distribution, the Thomson scattering amplitudes of a free electron are multiplied by the real form factor

$$
f(q)=4 \pi \int_{0}^{\infty} \rho(r) \frac{\sin (q r)}{q r} r^{2} d r
$$

where $\rho(r)$ is the electron charge distribution normalized such that

$$
4 \pi \int_{0}^{\infty} \rho(r) r^{2} d r=N
$$

where $N$ is the number of electrons in the atom. In form-factor approximation, the Rayleigh amplitudes are

$$
\begin{aligned}
& A_{\perp}^{R}=-r_{0} f(q), \\
& A_{\|}^{R}=A_{\perp}^{R} \cos \Theta .
\end{aligned}
$$


The form-factor approximation has also been derived in nonrelativistic and relativistic quantum mechanics. Extensive tabulations of the nonrelativistic form factor (NFF) (Ref. 10) and relativistic form factor (RFF) (Ref. 11 ) are readily available for neutral atoms $Z=1-100$. The form-factor approximation for elastic scattering is valid when the photon energy is large $(\hbar \omega \gg>T, T$ is the binding energy), as long as the momentum transfer is small $(\hbar q<<m c)$ and the electron binding energy is small $\left(T \ll m c^{2}\right)$, even for relativistic photon energies.

Franz ${ }^{12}$ has suggested the use of a modified relativistic form factor (MRFF)

$g_{i}(q)=4 \pi \int_{0}^{\infty} \rho_{i}(r) \frac{\sin (q r)}{(q r)}\left(\frac{m c^{2}}{E_{i}-V(r)}\right) r^{2} d r$,

where $\rho_{i}$ is the charge distribution and $E_{i}$ is the total energy (including rest mass energy) of the $i$ th atomic electron. $V(r)$ is the potential energy of a charge $e$ at position $r$. An extensive tabulation of MRFF values is readily available for neutral atoms $Z=1-100$ (Ref. 13). Generally, in the low-energy $\gamma$-ray region, the MRFF has been more successful than RFF and NFF in reproducing Rayleigh amplitudes predicted by the $S$-matrix method.

In order to improve the form-factor approximation, the dispersion relation is used to predict forward-angle corrections. Causality leads to analyticity for the forward scattering amplitude $A(\omega, 0)$ in the upper half of the complex $\omega$ plane. ${ }^{14}$ Consequently, one obtains dispersion relations such as

$$
\begin{aligned}
& \operatorname{Re} A(\omega, 0)=\frac{2 \omega^{2}}{\pi} \int_{0}^{\infty} \frac{\operatorname{Im} A\left(\omega^{\prime}, 0\right)}{\omega^{\prime}\left(\omega^{\prime 2}-\omega^{2}\right)} d \omega^{\prime}, \\
& \operatorname{Im} A(\omega, 0)=-\frac{2 \omega}{\pi} \int_{0}^{\infty} \frac{\operatorname{Re} A\left(\omega^{\prime}, 0\right)}{\omega^{\prime 2}-\omega^{2}} d \omega^{\prime},
\end{aligned}
$$

so that

$$
\begin{aligned}
& \operatorname{Re} A(\infty, 0)=-\frac{2}{\pi} \int_{0}^{\infty} \frac{\operatorname{Im} A\left(\omega^{\prime}, 0\right)}{\omega^{\prime}} d \omega^{\prime}, \\
& \operatorname{Im} A(\infty, 0)=0 .
\end{aligned}
$$

In nonrelativistic dipole approximation and formfactor approximation, $\operatorname{Re} A(\infty, 0)=-N r_{0}$. However, there are small relativistic corrections to $\operatorname{Re} A(\omega, 0)$; the MRFF gives these corrections. If, as is conventionally done, the anomalous scattering factors $f^{\prime}$ and $f^{\prime \prime}$ are defined by

$$
A(\omega, 0)=-r_{0}\left[f(0)+f^{\prime}(\omega)+i f^{\prime \prime}(\omega)\right],
$$

then

$f^{\prime}(\omega)=\left[-f(0)-\operatorname{Re} A(\infty, 0) / r_{0}\right]+\frac{2}{\pi} \int_{0}^{\infty} \frac{\omega^{\prime} f^{\prime \prime}\left(\omega^{\prime}\right)}{\omega^{\prime 2}-\omega^{2}} d \omega^{\prime}$.

Notice that the argument of $f(q)$ and $g(q)$ is the momentum transfer while that of $f^{\prime}(\omega)$ and $f^{\prime \prime}(\omega)$ is the photon energy. From the unitarity of the $S$ matrix (conservation of probability) follows the optical theorem

$$
\operatorname{Im} A(\omega, 0)=\frac{\omega}{4 \pi c} \sigma^{\text {tot }},
$$

relating the total cross section $\sigma^{\text {tot }}$ (including all elastic and inelastic processes) to the imaginary forward elastic scattering amplitude. This form of the optical theorem requires $\operatorname{Im} A(\omega, 0) \geq 0$ for all $\omega$. From the form of the dispersion relation, this implies that $\operatorname{Re} A(\infty, 0)<0$. This sign convention is different from that commonly used by crystallographers.

Cromer and Liberman ${ }^{15}$ used the optical theorem and dispersion relation together with theoretical photoeffect cross sections to estimate forward-angle anomalous scattering factors $f^{\prime}$ and $f^{\prime \prime}$ for x-ray energies. Their tabulation and associated computer code FPRIME (Ref. 16) are the most commonly used sources of $\mathrm{x}$-ray anomalous scattering factors. However, they used dipole approximation in the derivation of their results. While the dipole approximation is appropriate for the $\mathrm{x}$-ray energies of interest to them, they also used dipole approximation to compute the high-energy limit of $f^{\prime}$ as

$$
f_{\mathrm{CL}}^{\prime}(\infty)=\frac{5}{3} \frac{E_{\mathrm{tot}}}{m c^{2}},
$$

where $E_{\text {tot }}$ is the total ground-state energy of the atom. This use of dipole approximation to compute the highenergy limit results in an error that affects their $f^{\prime}$ values at all energies (see, e.g., Ref. 17).

It has been found that the high-energy limit of $S$ matrix theory is very accurately predicted by MRFF. Consequently,

$$
f_{\mathrm{SM}}^{\prime}(\infty) \approx-f(0)+g(0)
$$

So, improved predictions from anomalous scattering factors of Cromer and Liberman can be expected if their dipole-approximation high-energy limit is corrected with a more accurate value. We define a corrected real anomalous scattering factor as

$$
f_{\mathrm{CCL}}^{\prime}(\omega)=f_{\mathrm{CL}}^{\prime}(\omega)+\delta f^{\prime},
$$

where

$$
\delta f^{\prime}=-f_{\mathrm{CL}}^{\prime}(\infty)+f_{\mathrm{SM}}^{\prime}(\infty) .
$$

Similarly, an anomalous scattering factor for use with the MRFF could be defined as

$$
g_{\mathrm{CCL}}^{\prime}(\omega)=f_{\mathrm{CL}}^{\prime}(\omega)+\delta g^{\prime},
$$

where

$$
\delta g^{\prime}=-f_{\mathrm{CL}}^{\prime}(\infty)
$$

Table I lists some high-energy-limit values for photon scattering by noble-gas atoms. Tables II and III list anomalous scattering factors for krypton and xenon at photon energies of interest in this work. The so-called Jensen term ${ }^{18}$ is neglected in these values of $f^{\prime}$. The Jensen term is just one component of the high-energylimit correction to $f_{C L}^{\prime}$.

All derivations of $f^{\prime}(\omega)$ and $f^{\prime \prime}(\omega)$ have been for forward-angle scattering. While some authors have assumed that the angular dependence of the anomalous scattering factors would be given by the angular dependence of the form factor, comparison with $S$-matrix pre- 
TABLE I. High-energy-limit values for photon scattering by noble-gas atoms.

\begin{tabular}{rrrrrrr}
\hline \hline Atom & $f(0)$ & $g(0)$ & $f_{\mathrm{CL}}^{\prime}(\infty)$ & $f_{\mathrm{SM}}^{\prime}(\infty)$ & $\delta f^{\prime}$ & $\delta g^{\prime}$ \\
\hline${ }_{2} \mathrm{He}$ & 2 & 2.000 & -0.000 & -0.000 & 0.000 & 0.000 \\
${ }_{10} \mathrm{Ne}$ & 10 & 9.993 & -0.011 & -0.007 & 0.004 & 0.011 \\
${ }_{18} \mathrm{Ar}$ & 18 & 17.972 & -0.047 & -0.028 & 0.019 & 0.047 \\
${ }_{36} \mathrm{Kr}$ & 36 & 35.859 & -0.247 & -0.141 & 0.106 & 0.247 \\
${ }_{5}^{54} \mathrm{Xe}$ & 54 & 53.634 & -0.660 & -0.366 & 0.294 & 0.660 \\
${ }_{86} \mathrm{Rn}$ & 86 & 84.882 & -2.095 & -1.118 & 0.977 & 2.095 \\
\hline \hline
\end{tabular}

dictions indicates that this is not the case. In fact, comparisons with $S$-matrix predictions show that the angular dependence of $f^{\prime}, f^{\prime \prime}$ is much weaker than that predicted by the form factor. Currently there does not exist a good model for the angular dependence of $f^{\prime}$ and $f^{\prime \prime}$ and we will assume no angular dependence (aside from the explicit $\cos \Theta$ dependence in $A_{\|}$) in our predictions. So, we use high-energy-limit-corrected anomalousscattering-factor Rayleigh amplitudes of the form

$$
\begin{aligned}
& A_{\|}^{R}=-r_{0}\left[f(q)+f^{\prime}(\omega)+i f^{\prime \prime}(\omega)\right] \cos \Theta, \\
& A_{\perp}^{R}=-r_{0}\left[f(q)+f^{\prime}(\omega)+i f^{\prime \prime}(\omega)\right] .
\end{aligned}
$$

Tables IV and V compare various anomalousscattering-factor and form-factor predictions with $S$ matrix theory for polarized scattering by xenon. The energies considered are the maximum and minimum energies of these experiments, as well as the two energies closest to the xenon $K$-shell threshold. As can be seen from these comparisons, the high-energy-limit-corrected anomalous-scattering-factor predictions (labeled RFF + CCL and MRFF + CCL) are in the best agreement with $S$-matrix theory (labeled SM). Also, the high-energy-limit-corrected anomalous-scattering-factor predictions are a significant improvement over the uncorrected anomalous-scattering-factor predictions (labeled RFF $+\mathrm{CL}$ ), especially at larger angles. In addition, the $\mathrm{RFF}+\mathrm{CCL}$ and $\mathrm{MRFF}+\mathrm{CCL}$ predictions take a much smaller amount of computer resources to evaluate (measured in CPU seconds) as compared with the SM predictions (measured in CPU days).

\section{EXPERIMENT}

Synchrotron radiation from the electron storage ring DORIS II at Deutsches Elektronen-Synchrotron (DESY), Hamburg, was used for the experiments. A double Bragg monochromator shown schematically in Fig. 1, selects quasimonochromatic photons from the continuous spectrum of the SR. The two Ge(111) crystals of the monochromator are mounted on independent goniometer heads. ${ }^{19}$ The monochromatized beam passes through a beam defining aperture of $1-\mathrm{mm}$ diameter, and through a Compton polarimeter ${ }^{20}$ used for monitoring the intensity and measuring the linear polarization. After having traversed the scattering chamber, the beam is stopped in a shielded beam catcher.

The scattering chamber (12-cm diameter) is equipped with $50-\mu \mathrm{m}$ Kapton windows for the direct beam and for the scattered photons. A $100 \mathrm{~mm}^{2} \times 5 \mathrm{~mm}$ intrinsic $\mathrm{Ge}$ planar detector is located behind a collimator system, at a distance of $23 \mathrm{~cm}$ from the center of the scattering chamber. Its linewidth is 330 and $380 \mathrm{eV}$ full width at half maximum at 21 and $44 \mathrm{keV}$, respectively.

The scattering chamber contains the target gas, either $\mathrm{Kr}$ or $\mathrm{Xe}$, at room temperature and at a pressure of about 500 Torr. Both temperature and pressure are registered continuously, yielding the density of the target gas. The effective target volume is that part of the chamber volume which is traversed by the direct beam and viewed by the detector through its collimator system.

The assembly of scattering chamber and detector can be rotated around the axis of the direct beam. In this way the scattering plane can be oriented either parallel or perpendicular to the (horizontal) plane of polarization of the direct beam.

The Compton polarimeter was calibrated as an intensity monitor by placing the $\mathrm{Ge}$ detector into the direct beam after shielding it by a $\mathrm{Cu}$ absorber which reduces the intensity to a tolerable level. For several of the photon energies $E$ used in the experiments, the attenuation coefficient $\mu(E)$ of $\mathrm{Cu}$ was measured by varying the thickness of the absorber and recording the correspond-

TABLE II. Anomalous scattering factors at selected photon energies for krypton. CL indicates data from code of Cromer and Liberman. CCL indicates CL data with corrected high-energy limit. The sign of $f_{\mathrm{CL}}^{\prime \prime}$ has been changed to be consistent with the phase conventions used here.

\begin{tabular}{lcccc}
\hline \hline$E(\mathrm{keV})$ & $f_{\mathrm{CL}}^{\prime \prime}=f_{\mathrm{CCL}}^{\prime \prime}$ & $f_{\mathrm{CL}}^{\prime}$ & $f_{\mathrm{CCL}}^{\prime}=f_{\mathrm{Cl}}^{\prime}+\delta f^{\prime}$ & $g_{\mathrm{CCL}}^{\prime}=f_{\mathrm{CL}}^{\prime}+\delta g^{\prime}$ \\
\hline 21.2 & -1.964 & -0.059 & 0.047 & 0.188 \\
28.5 & -1.173 & 0.167 & 0.273 & 0.414 \\
31.2 & -0.998 & 0.172 & 0.278 & 0.419 \\
35.36 & -0.795 & 0.158 & 0.264 & 0.405 \\
43.7 & -0.536 & 0.104 & 0.210 & 0.351 \\
\hline \hline
\end{tabular}


TABLE III. Anomalous scattering factors at selected photon energies for xenon. CL indicates data from code of Cromer and Liberman. CCL indicates CL data with corrected high-energy limit. The sign of $f_{\mathrm{Cl}}^{\prime \prime}$ has been changed to be consistent with the phase conventions used here.

\begin{tabular}{lcccc}
\hline \hline$E(\mathrm{keV})$ & $f_{\mathrm{CL}}^{\prime \prime}=f_{\mathrm{CCL}}^{\prime \prime}$ & $f_{\mathrm{CL}}^{\prime}$ & $f_{\mathrm{CCL}}^{\prime}=f_{\mathrm{CL}}^{\prime}+\delta f^{\prime}$ & $g_{\mathrm{CCL}}^{\prime}=f_{\mathrm{CL}}^{\prime}+\delta g^{\prime}$ \\
\hline 21.2 & -1.390 & -1.002 & -0.708 & -0.342 \\
28.5 & -0.815 & -1.718 & -1.424 & -1.058 \\
31.2 & -0.690 & -2.183 & -1.889 & -1.523 \\
33.95 & -0.590 & -3.592 & -3.298 & -2.932 \\
34.26 & -0.581 & -4.215 & -3.921 & -3.555 \\
34.42 & -0.576 & -4.898 & -4.604 & -4.238 \\
35.36 & -3.377 & -3.156 & -2.862 & -2.496 \\
39.8 & -2.785 & -1.285 & -0.991 & -0.625 \\
43.7 & -2.387 & -0.810 & -0.516 & -0.150 \\
\hline \hline
\end{tabular}

ing changes in the transmission factor. The tabulation of photon cross sections by Plechaty and Terrall ${ }^{21}$ was found to agree within $0.5 \%$, on the average, with the measured values of $\mu(E)$. The uncertainty of $\mu(E)$ contributes a large part of the total experimental error of the results. A further source of error is the presence of photons having energies $3 E, 4 E, \ldots$ in the attenuated direct beam. These photons correspond to harmonics of the fundamental energy $E$ selected by the monochromator. Photons of energy $2 E$ are not reflected by the Ge(111) planes. While the transmission factors of the $\mathrm{Cu}$ absorbers for the fundamental energy $E$ varied between $3 \times 10^{-6}$ and $2 \times 10^{-5}$ at $E=21.2$ and $43.7 \mathrm{keV}$, respectively, the transmission factors for the harmonics were always larger than 0.3 . Therefore, a very small relative contribution of harmonics in the monochromatized beam appears vastly enhanced after attenuation by the $\mathrm{Cu}$ absorber. As a consequence, pile-up and dead-time losses make the determination of the intensity of the direct beam difficult and, in extreme cases, impossible. The relative contribution of harmonics in the direct beam was reduced by (i) rotating the second monochromator crystal slightly away from exact parallelism to the first one, and (ii) performing the experiments at as low an energy $E_{\mathrm{el}}$ of the orbiting electrons as possible. Since DORIS II is operated either at $E_{\mathrm{el}}=3.5 \mathrm{GeV}$ (critical energy of the SR spectrum $E_{c}=7.8 \mathrm{keV}$ ) or at $E_{\mathrm{el}}=5$ $\mathrm{GeV}\left(E_{c}=22.8 \mathrm{keV}\right)$, the experiments at $E$ below and above $35 \mathrm{keV}$ were performed at $E_{\mathrm{el}}=3.5$ and $5 \mathrm{GeV}$, respectively. At photon energies below $20 \mathrm{keV}$ the direct beam could not be measured due to the problems mentioned above. For energies $E$ above $44 \mathrm{keV}$ the Bragg angles were below the mechanical limit of the monochromator.

The spectra of scattered photons do not show any contribution of higher harmonics of $E$ since the unattenuated direct beam is used for scattering. In the case of $\mathrm{Xe}$ at photon energies $E$ above the $K$-shell binding energy $B_{K}=34.561 \mathrm{keV}, K-X$ fluorescence is excited. Its large intensity prevents determination of scattering cross sections at energies $B_{K} \leq E<35.4 \mathrm{keV}$.

In converting the count rate of scattered photons to a differential cross section, the geometry of the experiment and the attenuation of the incident as well as of the scattered photons in the target gas were taken into account.
This was done by subdividing the effective target volume into a large number of volume elements and calculating the contributions of the elements to the count rate of scattered photons.

\section{RESULTS AND DISCUSSION}

Spectra of scattered photons were measured at energies of the incident photons in the interval $E=21.2-43.7 \mathrm{keV}$. The scattering angles were $\Theta=60^{\circ}$, $90^{\circ}$, and $120^{\circ}$ in the vertical $(v)$, and $\Theta=60^{\circ}$ and $120^{\circ}$ in the horizontal $(h)$ scattering plane. The number of elastically scattered photons was normalized to the number of counts in the Compton polarimeter. In this way the efficiency of the detector is eliminated from the evaluation of cross sections. Simultaneously, the degree of polarization $P_{v}$ or $P_{h}$ of the direct beam was measured. Both the intensity and the polarization of the direct beam were registered continuously during each scattering run because they are influenced by any variation of the electron beam in the storage ring. Values of $P_{v}$ or $P_{h}$ were in the intervals $P=0.85-0.95$ and $P=0.82-0.9$ at $E_{\mathrm{el}}=3.5$ and $5 \mathrm{GeV}$, respectively. The variation of $P$ was within a few percent during a single scattering run, the duration of which was limited by the lifetime of the electron beam in the storage ring. The total recording time used for measuring a cross section varied between 600 and $12000 \mathrm{~s}$, depending on the intensity of the direct beam, the photon energy, and the scattering angle.

The evaluation yields effective differential scattering cross sections $d \sigma_{v} / d \Omega$ and $d \sigma_{h} / d \Omega$. At $\Theta=60^{\circ}$ and $120^{\circ}$, one obtains the experimental differential cross sec-

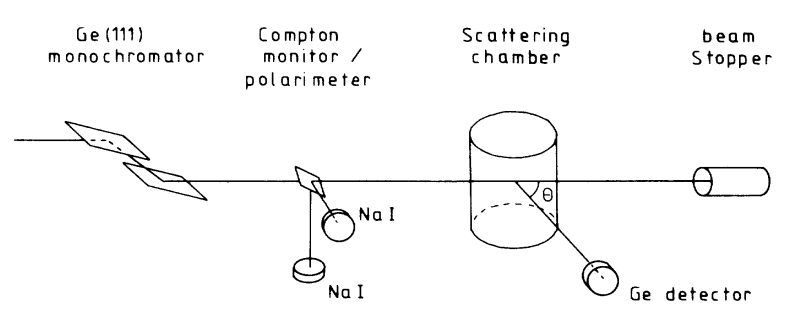

FIG. 1. Experimental apparatus. The NaI detectors of the Compton polarimeter are oriented at right angles to each other as well as to the photon beam. 
TABLE IV. Comparison of $S$-matrix predictions with various form-factor theories for scattering of perpendicular-polarized photons by xenon; SM is the $S$-matrix theory; RFF is the relativistic form factor; MRFF is the modified relativistic form factor; NFF is the nonrelativistic form factor; $C L$ is the angle-independent, uncorrected, anomalous scattering factors; CCL is the angleindependent, high-energy-limit-corrected, anomalous scattering factors. The small contribution due to the nuclear Thomson amplitude is included. Numbers in square brackets are the associated power of 10 .

\begin{tabular}{|c|c|c|c|c|c|c|c|c|}
\hline \multirow[b]{2}{*}{$E(\mathrm{keV})$} & \multirow[b]{2}{*}{$\Theta(\mathrm{deg})$} & \multirow[b]{2}{*}{ SM } & \multirow[b]{2}{*}{$\mathrm{RFF}+\mathrm{CCL}$} & \multicolumn{2}{|c|}{$d \sigma_{\perp} / d \Omega\left(r_{0}^{2} / \mathrm{sr}\right)$} & \multirow[b]{2}{*}{ MRFF } & \multirow[b]{2}{*}{ NFF } & \multirow[b]{2}{*}{ RFF } \\
\hline & & & & $\mathrm{MRFF}+\mathrm{CCL}$ & $\mathrm{RFF}+\mathrm{CL}$ & & & \\
\hline \multirow[t]{5}{*}{21.2} & 0 & $2.84[3]$ & $2.84[3]$ & $2.84[3]$ & $2.81[3]$ & $2.88[3]$ & $2.92[3]$ & $2.92[3]$ \\
\hline & 60 & $3.62[2]$ & $3.65[2]$ & $3.66[2]$ & $3.54[2]$ & $3.77[2]$ & $3.77[2]$ & $3.90[2]$ \\
\hline & 90 & $2.14[2]$ & $2.16[2]$ & $2.17[2]$ & $2.08[2]$ & $2.25[2]$ & $2.27[2]$ & $2.35[2]$ \\
\hline & 120 & $1.44[2]$ & $1.46[2]$ & $1.47[2]$ & $1.39[2]$ & $1.53[2]$ & $1.54[2]$ & $1.61[2]$ \\
\hline & 180 & $9.93[1]$ & $1.02[2]$ & $1.02[2]$ & $9.58[1]$ & $1.07[2]$ & $1.08[2]$ & $1.14[2]$ \\
\hline \multirow[t]{5}{*}{34.42} & 0 & $2.44[3]$ & $2.44[3]$ & $2.43[3]$ & $2.41[3]$ & $2.88[3]$ & $2.92[3]$ & $2.92[3]$ \\
\hline & 60 & $8.28[1]$ & $8.05[1]$ & $7.97[1]$ & $7.53[1]$ & $1.75[2]$ & $1.77[2]$ & $1.84[2]$ \\
\hline & 90 & $1.95[1]$ & $1.80[1]$ & $1.81[1]$ & $1.56[1]$ & $7.30[1]$ & $7.26[1]$ & $7.76[1]$ \\
\hline & 120 & 5.12 & 4.64 & 4.60 & 3.51 & $4.09[1]$ & $4.10[1]$ & $4.46[1]$ \\
\hline & 180 & 1.69 & 1.47 & 1.46 & $9.27[-1]$ & $2.91[1]$ & $3.10[1]$ & $3.21[1]$ \\
\hline \multirow[t]{5}{*}{35.36} & 0 & $2.63[3]$ & $2.63[3]$ & $2.63[3]$ & $2.60[3]$ & $2.88[3]$ & $2.92[3]$ & $2.92[3]$ \\
\hline & 60 & $1.20[2]$ & $1.18[2]$ & $1.19[2]$ & $1.12[2]$ & $1.65[2]$ & $1.67[2]$ & $1.74[2]$ \\
\hline & 90 & $4.32[1]$ & $4.32[1]$ & $4.40[1]$ & $4.00[1]$ & $6.74[1]$ & $6.71[1]$ & $7.23[1]$ \\
\hline & 120 & $2.35[1]$ & $2.44[1]$ & $2.48[1]$ & $2.24[1]$ & $3.79[1]$ & $3.82[1]$ & 4.19[1] \\
\hline & 180 & $1.73[1]$ & $1.84[1]$ & $1.90[1]$ & $1.69[1]$ & $2.76[1]$ & $2.97[1]$ & $3.03[1]$ \\
\hline \multirow[t]{5}{*}{43.7} & 0 & $2.87[3]$ & $2.87[3]$ & $2.87[3]$ & $2.84[3]$ & $2.88[3]$ & $2.92[3]$ & $2.92[3]$ \\
\hline & 60 & $1.02[2]$ & $1.00[2]$ & $1.02[2]$ & $9.46[1]$ & $9.92[1]$ & $9.92[1]$ & $1.05[2]$ \\
\hline & 90 & $4.02[1]$ & $4.03[1]$ & $4.10[1]$ & $3.69[1]$ & $3.72[1]$ & $3.74[1]$ & $4.10[1]$ \\
\hline & 120 & $2.71[1]$ & $2.75[1]$ & $2.84[1]$ & $2.49[1]$ & $2.42[1]$ & $2.63[1]$ & $2.69[1]$ \\
\hline & 180 & $2.16[1]$ & $2.20[1]$ & $2.30[1]$ & $1.97[1]$ & $1.86[1]$ & $1.87[1]$ & $2.07[1]$ \\
\hline
\end{tabular}

tions for linearly polarized photons via

$d \sigma_{\perp}^{\mathrm{expt}} / d \Omega=\frac{\left(1+Q_{v}\right) d \sigma_{v} / d \Omega-\left(1+Q_{h}\right) Q_{v} d \sigma_{h} / d \Omega}{1-Q_{v} Q_{h}}$

and an analogous expression for $d \sigma_{\|}^{\operatorname{expt}} / d \Omega$. Here, $Q_{v(h)}=\left(1-P_{v(h)}\right) /\left(1+P_{v(h)}\right) . \quad$ At $\quad \Theta=90^{\circ}$, where $d \sigma_{h} / d \Omega$ was not measured, $d \sigma_{\|} / d \Omega$ was assumed to be negligibly small, and the evaluation yields $d \sigma_{1}^{\text {expt }} / d \Omega=\left(1+Q_{v}\right) d \sigma_{v} / d \Omega$. A similar procedure was used at $E=33.95,34.26$, and $34.42 \mathrm{keV}$, where only scattering by $\mathrm{Xe}$ in the $v$ plane was evaluated. Here, $d \sigma_{\perp}^{\text {expt }} / d \Omega$ was calculated using the measured values of $d \sigma_{v} / d \Omega$ and $P_{v}$ and, in addition, the theoretical $S$ matrix predictions $d \sigma_{\|}^{\mathrm{SM}} / d \Omega$. This seems to be justified since, for $P_{v}>0.85$, the resulting $d \sigma_{\perp}^{\operatorname{expt}} / d \Omega$ does not depend sensitively on the value of $d \sigma_{\|} / d \Omega$ used in the evaluation.

At $E=33.95,34.26$, and $34.42 \mathrm{keV}$, resonant Raman scattering (RRS) contributes to the elastic peak of Xe since the detector does not resolve RRS from elastic scattering if the final state of the former process contains a hole in the $N$ or $O$ shell of the scattering atom. The contribution of RRS was calculated using the theory

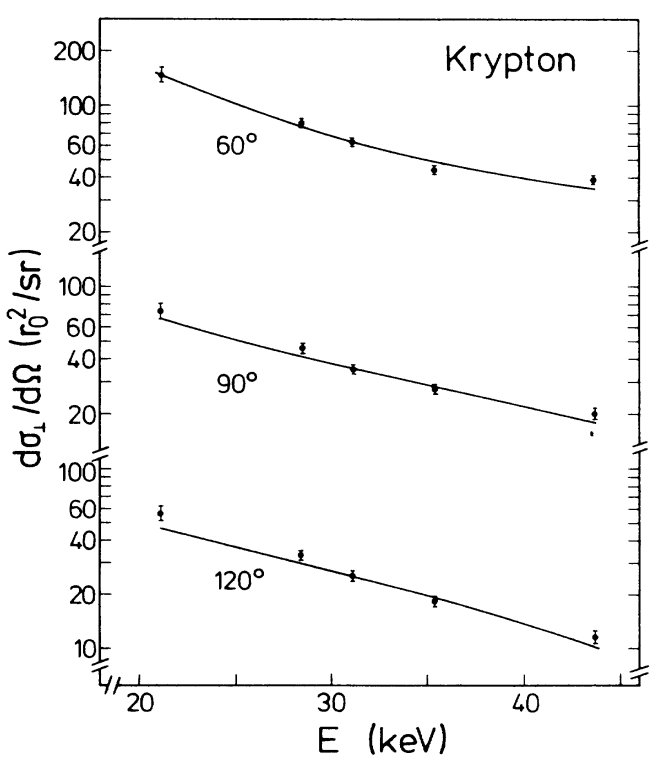

FIG. 2. Differential cross sections for elastic scattering of linearly polarized photons by $\mathrm{Kr}$ atoms. Points, experimental results; curves, theoretical SM predictions. 
TABLE V. Comparison of $S$-matrix predictions with various form-factor theories for scattering of parallel-polarized photons by xenon; SM is the $S$-matrix theory; RFF is the relativistic form factor; MRFF is the modified relativistic form factor; NFF is the nonrelativistic form factor; $\mathrm{CL}$ is the angle-independent uncorrected, anomalous scattering factors; CCL is the angle-independent, high-energy-limited-corrected, anomalous scattering factors. The small contribution due to the nuclear Thomson amplitude is included. Numbers in square brackets are the associated power of 10.

\begin{tabular}{|c|c|c|c|c|c|c|c|c|}
\hline \multirow[b]{2}{*}{$E(\mathrm{keV})$} & \multirow[b]{2}{*}{$\Theta(\operatorname{deg})$} & \multicolumn{7}{|c|}{$d \sigma_{\|} / d \Omega\left(r_{0}^{2} / \mathrm{sr}\right)$} \\
\hline & & SM & $\mathrm{RFF}+\mathrm{CCL}$ & $\mathrm{MRFF}+\mathrm{CCL}$ & $\mathrm{RFF}+\mathrm{CL}$ & MRFF & NFF & RFF \\
\hline \multirow[t]{5}{*}{21.2} & 0 & $2.84[3]$ & $2.84[3]$ & $2.84[3]$ & $2.82[3]$ & $2.88[3]$ & $2.92[3]$ & $2.92[3]$ \\
\hline & 60 & $8.87[1]$ & $9.12[1]$ & $9.14[1]$ & $8.84[1]$ & $9.42[1]$ & $9.42[1]$ & $9.76[1]$ \\
\hline & 90 & $1.79[-2]$ & 0 & 0 & 0 & 0 & 0 & 0 \\
\hline & 120 & $3.69[1]$ & $3.64[1]$ & $3.66[1]$ & $3.46[1]$ & $3.82[1]$ & $3.85[1]$ & $4.03[1]$ \\
\hline & 180 & $9.93[1]$ & $1.02[2]$ & $1.02[2]$ & $9.58[1]$ & $1.07[2]$ & $1.08[2]$ & $1.14[2]$ \\
\hline \multirow[t]{5}{*}{34.42} & 0 & $2.44[3]$ & $2.44[3]$ & $2.43[3]$ & $2.41[3]$ & $2.88[3]$ & $2.92[3]$ & $2.92[3]$ \\
\hline & 60 & $2.05[1]$ & $2.01[1]$ & $1.99[1]$ & $1.88[1]$ & $4.38[1]$ & $4.42[1]$ & $4.59[1]$ \\
\hline & 90 & $2.28[-3]$ & 0 & 0 & 0 & 0 & 0 & 0 \\
\hline & 120 & 1.28 & 1.16 & 1.15 & $8.77[-1]$ & $1.02[1]$ & $1.02[1]$ & $1.12[1]$ \\
\hline & 180 & 1.69 & 1.47 & 1.46 & $9.27[-1]$ & $2.91[1]$ & $3.10[1]$ & $3.21[1]$ \\
\hline \multirow[t]{5}{*}{35.36} & 0 & $2.63[3]$ & $2.63[3]$ & $2.62[3]$ & $2.60[3]$ & $2.88[3]$ & $2.92[3]$ & $2.92[3]$ \\
\hline & 60 & $2.91[1]$ & $2.95[1]$ & $2.97[1]$ & $2.80[1]$ & $4.13[1]$ & $4.18[1]$ & $4.34[1]$ \\
\hline & 90 & $9.32[-3]$ & 0 & 0 & 0 & 0 & 0 & 0 \\
\hline & 120 & 6.17 & 6.11 & 6.20 & 5.60 & 9.48 & 9.56 & $1.05[1]$ \\
\hline & 180 & $1.73[1]$ & $1.84[1]$ & $1.90[1]$ & $1.69[1]$ & $2.76[1]$ & $2.97[1]$ & $3.03[1]$ \\
\hline \multirow[t]{5}{*}{43.7} & 0 & $2.87[3]$ & $2.87[3]$ & $2.87[3]$ & $2.84[3]$ & $2.88[3]$ & $2.92[3]$ & $2.92[3]$ \\
\hline & 60 & $2.46[1]$ & $2.51[1]$ & $2.55[1]$ & $2.36[1]$ & $2.48[1]$ & $2.48[1]$ & $2.62[1]$ \\
\hline & 90 & $1.61[-2]$ & 0 & 0 & 0 & 0 & 0 & 0 \\
\hline & 120 & 7.15 & 6.88 & 7.10 & 6.22 & 6.04 & 6.57 & 6.73 \\
\hline & 180 & $2.16[1]$ & $2.20[1]$ & $2.30[1]$ & $1.97[1]$ & $1.86[1]$ & $1.87[1]$ & $2.07[1]$ \\
\hline
\end{tabular}

outlined in Ref. 22 and subtracted before evaluating the elastic cross section. This procedure is justified because the theory has been checked experimentally and found valid within $5 \%$ (Ref. 22).

Tables VI-IX list the experimental results together with theoretical predictions. The theoretical predictions include contributions from Rayleigh $(R)$ and nuclear Thomson $(T)$ amplitudes. In Figs. 2 and 3 the experimental results and the SM predictions are plotted together.

In general, the experimental values agree well with the $\mathrm{SM}, \mathrm{RFF}+\mathrm{CCL}$, and MRFF + CCL predictions of $d \sigma_{\perp} / d \Omega$. Especially at photon energies close to the $K$ shell binding energy of $\mathrm{Xe}$, very good agreement between theory and experiment is found. Only at the smallest and the largest photon energies used in the experiments are the differences between experiment and theory comparable to, or slightly larger than, the experimental errors. However, at these energies the direct-beam calibration of the Compton polarimeter was quite difficult, possibly causing additional systematic errors of the order of $5 \%$ at these energies.

Experiment does not generally distinguish between the $\mathrm{SM}, \mathrm{RFF}+\mathrm{CCL}$, and MRFF + CCL theories. However, the RFF + CL, MRFF, NFF, and RFF theoretical predictions are clearly inferior. In the case of $\mathrm{Kr}$, the RFF and MRFF cross sections are, on the average, below the SM values by $3 \%$ and $7 \%$, respectively. In

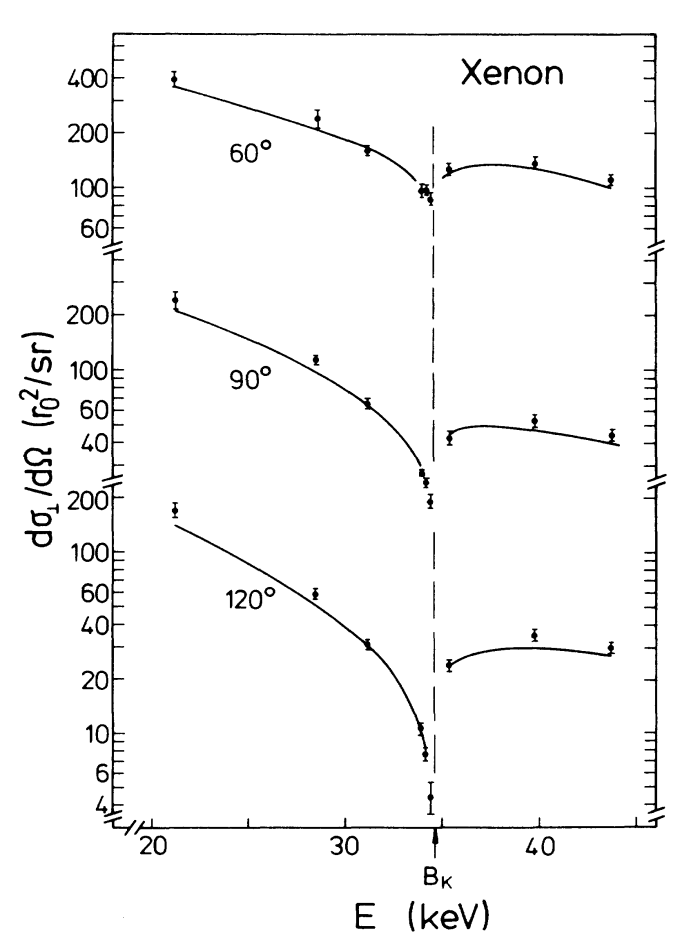

FIG. 3. Same as Fig. 2, but for scattering by Xe atoms. $B_{K}$ denotes the $K$-shell binding energy of $\mathrm{Xe}$. 
TABLE VI. Comparison of experiment and theory for scattering of perpendicular-polarized photons by krypton; SM is the $S$-matrix theory; RFF is the relativistic form factor; MRFF is the modified relativistic form factor, $\mathrm{CCL}$ is the angle-dependent, high-energy-limit-corrected, anomalous scattering factors. The small contribution due to the nuclear Thomson amplitude is included. Numbers in square brackets are the associated power of 10 .

\begin{tabular}{|c|c|c|c|c|c|}
\hline \multirow[b]{2}{*}{$E(\mathrm{keV})$} & \multirow[b]{2}{*}{$\Theta(\operatorname{deg})$} & \multicolumn{4}{|c|}{$d \sigma_{\perp} / d \Omega\left(r_{0}^{2} / \mathrm{sr}\right)$} \\
\hline & & Experiment & SM & $\mathrm{RFF}+\mathrm{CCL}$ & $\mathrm{MRFF}+\mathrm{CCL}$ \\
\hline \multirow[t]{3}{*}{21.2} & 60 & $1.48 \pm 0.13[2]$ & $1.44[2]$ & $1.43[2]$ & $1.43[2]$ \\
\hline & 90 & $7.4 \pm 0.7[1]$ & $6.71[1]$ & $6.69[1]$ & $6.72[1]$ \\
\hline & 120 & $5.7 \pm 0.5[1]$ & $4.69[1]$ & $4.67[1]$ & $4.68[1]$ \\
\hline \multirow[t]{3}{*}{28.5} & 60 & $7.79 \pm 0.37[1]$ & $7.48[1]$ & $7.54[1]$ & $7.61[1]$ \\
\hline & 90 & $4.57 \pm 0.21[1]$ & $4.05[1]$ & $4.07[1]$ & $4.13[1]$ \\
\hline & 120 & $3.33 \pm 0.16[1]$ & $3.00[1]$ & $3.04[1]$ & $3.07[1]$ \\
\hline \multirow[t]{3}{*}{31.2} & 60 & $6.21 \pm 0.31[1]$ & $6.20[1]$ & $6.26[1]$ & $6.27[1]$ \\
\hline & 90 & $3.50 \pm 0.14[1]$ & $3.53[1]$ & $3.55[1]$ & $3.59[1]$ \\
\hline & 120 & $2.54 \pm 0.10[1]$ & $2.54[1]$ & $2.58[1]$ & $2.60[1]$ \\
\hline \multirow[t]{3}{*}{35.36} & 60 & $4.43 \pm 0.22[1]$ & $4.86[1]$ & $4.90[1]$ & $4.95[1]$ \\
\hline & 90 & $2.73 \pm 0.12[1]$ & $2.84[1]$ & $2.89[1]$ & $2.92[1]$ \\
\hline & 120 & $1.85 \pm 0.08[1]$ & $1.91[1]$ & $1.94[1]$ & $1.98[1]$ \\
\hline \multirow[t]{3}{*}{43.7} & 60 & $3.87 \pm 0.18[1]$ & $3.41[1]$ & $3.45[1]$ & $3.49[1]$ \\
\hline & 90 & $2.02 \pm 0.13[1]$ & $1.80[1]$ & $1.82[1]$ & $1.86[1]$ \\
\hline & 120 & $1.17 \pm 0.08[1]$ & $1.03[1]$ & $1.06[1]$ & $1.09[1]$ \\
\hline
\end{tabular}

the case of $\mathrm{Xe}$, similar differences are found at $E=21.2-31.2 \mathrm{keV}$. At $E=35.36-43.7 \mathrm{keV}$, i.e., above the $K$-shell binding energy, the respective differences are larger, being $9 \%$ and $15 \%$. No systematic behavior is found at $E=33.95$ and $34.26 \mathrm{keV}$. As mentioned earlier, the anomalous scattering factors $f_{\mathrm{CCL}}^{\prime}, g_{\mathrm{CCL}}^{\prime}$ and $f_{\mathrm{CCL}}^{\prime \prime}$ are taken to be independent of $\Theta$, and the Jensen contri- bution $^{18}$ to $f^{\prime}$ is omitted. Scaling of the anomalous scattering factors with the angular dependence of $f(q)$ or $g(q)$ makes the predictions even worse, as does the inclusion of the Jensen term in $f^{\prime}$.

The incomplete linear polarization of the direct beam limits the precision of determining $d \sigma_{\|}^{\exp } / d \Omega$. Since $d \sigma_{\perp} / d \Omega$ is large in comparison to $d \sigma_{\|} / d \Omega$, a small con-

TABLE VII. Comparison of experiment and theory for scattering of parallel-polarized photons by krypton, SM is the $S$-matrix theory; RFF is the relativistic form factor; MRFF is the modified relativistic form factor; CCL is the angle-independent, high-energy-limit-corrected, anomalous scattering factors. The small contribution due to the nuclear Thomson amplitude is included. Numbers in square brackets are the associated power of 10 .

\begin{tabular}{|c|c|c|c|c|c|}
\hline \multirow[b]{2}{*}{$E(\mathrm{keV})$} & \multirow[b]{2}{*}{$\theta(\operatorname{deg})$} & \multicolumn{4}{|c|}{$d \sigma_{\|} / d \Omega\left(r_{0}^{2} / \mathrm{sr}\right)$} \\
\hline & & Experiment & SM & $\mathrm{RFF}+\mathrm{CCL}$ & $\mathrm{MRFF}+\mathrm{CCL}$ \\
\hline \multirow[t]{3}{*}{21.2} & 60 & $4.1 \pm 1.6[1]$ & $3.56[1]$ & $3.57[1]$ & $3.58[1]$ \\
\hline & 90 & & $3.68[-3]$ & 0 & 0 \\
\hline & 120 & $1.4 \pm 0.6[1]$ & $1.19[1]$ & $1.17[1]$ & $1.17[1]$ \\
\hline \multirow[t]{3}{*}{28.5} & 60 & $2.1 \pm 0.9[1]$ & $1.85[1]$ & $1.89[1]$ & $1.90[1]$ \\
\hline & 90 & & $3.29[-3]$ & 0 & 0 \\
\hline & 120 & $7 \pm 4$ & 7.63 & 7.60 & 7.66 \\
\hline \multirow[t]{3}{*}{31.2} & 60 & $1.8 \pm 0.6[1]$ & $1.54[1]$ & $1.57[1]$ & $1.57[1]$ \\
\hline & 90 & & $2.60[-3]$ & 0 & 0 \\
\hline & 120 & $7 \pm 2$ & 6.44 & 6.45 & 6.50 \\
\hline \multirow[t]{3}{*}{35.36} & 60 & $2.0 \pm 0.8[1]$ & $1.20[1]$ & $1.23[1]$ & $1.24[1]$ \\
\hline & 90 & & $2.92[-3]$ & 0 & 0 \\
\hline & 120 & $8 \pm 4$ & 4.87 & 4.85 & 4.96 \\
\hline \multirow[t]{3}{*}{43.7} & 60 & $9 \pm 4$ & 8.38 & 8.62 & 8.73 \\
\hline & 90 & & $2.88[-3]$ & 0 & 0 \\
\hline & 120 & $3 \pm 1$ & 2.66 & 2.65 & 2.72 \\
\hline
\end{tabular}


TABLE VIII. Comparison of experiment and theory for scattering of perpendicular-polarized photons by xenon; SM is the $S$ matrix theory; RFF is the relativistic form factor; MRFF is the modified relativistic form factor; CCL is the angle-independent, high-energy-limit-corrected, anomalous scattering factors. The small contribution due to the nuclear Thomson amplitude is included. Numbers in square brackets are the associated power of 10 .

\begin{tabular}{|c|c|c|c|c|c|}
\hline \multirow[b]{2}{*}{$E(\mathrm{keV})$} & \multirow[b]{2}{*}{$\theta(\mathrm{deg})$} & \multicolumn{4}{|c|}{$d \sigma_{1} / d \Omega\left(r_{0}^{2} / \mathbf{s r}\right)$} \\
\hline & & Experiment & SM & $\mathrm{RFF}+\mathrm{CCL}$ & $\mathrm{MRFF}+\mathrm{CCL}$ \\
\hline \multirow[t]{3}{*}{21.2} & 60 & $3.93 \pm 0.36[2]$ & $3.62[2]$ & $3.65[2]$ & $3.66[2]$ \\
\hline & 90 & $2.41 \pm 0.23[2]$ & $2.14[2]$ & $2.16[2]$ & $2.17[2]$ \\
\hline & 120 & $1.71 \pm 0.14[2]$ & $1.44[2]$ & $1.46[2]$ & $1.47[2]$ \\
\hline \multirow[t]{3}{*}{28.5} & 60 & $2.30 \pm 0.14[2]$ & $2.10[2]$ & $2.11[2]$ & $2.12[2]$ \\
\hline & 90 & $1.13 \pm 0.06[2]$ & $9.90[1]$ & $1.01[2]$ & $1.01[2]$ \\
\hline & 120 & $5.9 \pm 0.3[1]$ & $5.19[1]$ & $5.29[1]$ & $5.41[1]$ \\
\hline \multirow[t]{3}{*}{31.2} & 60 & $1.59 \pm 0.07[2]$ & $1.69[2]$ & $1.69[2]$ & $1.70[2]$ \\
\hline & 90 & $6.50 \pm 0.33[1]$ & $6.89[1]$ & $6.82[1]$ & $6.95[1]$ \\
\hline & 120 & $3.16 \pm 0.13[1]$ & $3.33[1]$ & $3.31[1]$ & $3.42[1]$ \\
\hline \multirow[t]{3}{*}{33.95} & 60 & $9.63 \pm 0.68[1]$ & $1.10[2]$ & $1.09[2]$ & $1.10[2]$ \\
\hline & 90 & $2.74 \pm 0.17[1]$ & $3.31[1]$ & $3.27[1]$ & $3.37[1]$ \\
\hline & 120 & $1.04 \pm 0.06[1]$ & $1.25[1]$ & $1.25[1]$ & $1.32[1]$ \\
\hline \multirow[t]{3}{*}{34.26} & 60 & $9.74 \pm 0.57[1]$ & $9.36[1]$ & $9.44[1]$ & $9.53[1]$ \\
\hline & 90 & $2.43 \pm 0.14[1]$ & $2.47[1]$ & $2.48[1]$ & $2.58[1]$ \\
\hline & 120 & $7.5 \pm 0.6$ & 7.88 & 8.14 & 8.64 \\
\hline \multirow[t]{3}{*}{34.42} & 60 & $8.70 \pm 0.63[1]$ & $8.28[1]$ & $8.05[1]$ & $7.97[1]$ \\
\hline & 90 & $1.90 \pm 0.17[1]$ & $1.95[1]$ & $1.80[1]$ & $1.81[1]$ \\
\hline & 120 & $4.4 \pm 0.9$ & 5.12 & 4.64 & 4.60 \\
\hline \multirow[t]{3}{*}{35.36} & 60 & $1.27 \pm 0.08[2]$ & $1.20[2]$ & $1.18[2]$ & $1.19[2]$ \\
\hline & 90 & $4.22 \pm 0.30[1]$ & $4.32[1]$ & $4.32[1]$ & $4.40[1]$ \\
\hline & 120 & $2.40 \pm 0.17[1]$ & $2.35[1]$ & $2.44[1]$ & $2.48[1]$ \\
\hline \multirow[t]{3}{*}{39.8} & 60 & $1.36 \pm 0.11[2]$ & $1.21[2]$ & $1.20[2]$ & $1.21[2]$ \\
\hline & 90 & $5.30 \pm 0.42[1]$ & $4.66[1]$ & $4.63[1]$ & $4.71[1]$ \\
\hline & 120 & $3.50 \pm 0.24[1]$ & $2.93[1]$ & $2.96[1]$ & $3.04[1]$ \\
\hline \multirow[t]{3}{*}{43.7} & 60 & $1.11 \pm 0.07[2]$ & $1.02[2]$ & $1.00[2]$ & $1.02[2]$ \\
\hline & 90 & $4.4 \pm 0.3[1]$ & $4.02[1]$ & $4.03[1]$ & $4.10[1]$ \\
\hline & 120 & $3.0 \pm 0.2[1]$ & $2.71[1]$ & $2.75[1]$ & $2.84[1]$ \\
\hline
\end{tabular}

tribution of vertically polarized photons in the direct beam provides a considerable contribution of $d \sigma_{\perp} / d \Omega$ to the photons scattered in the horizontal plane, i.e., to $d \sigma_{h} / d \Omega$. In addition, the statistical error of the scattered intensity is larger in the horizontal plane than in the vertical one, due to the lower total intensity of scattered photons.

\section{CONCLUSIONS}

The present work is the first experiment on Rayleigh scattering using hard synchrotron $x$ rays with $E>20$ $\mathrm{keV}$. For the first time, scattering cross sections were systematically investigated at photon energies close to the $K$-shell binding energy of the scattering atoms. Comparison of experimental results with theoretical predictions show that the numerical second-order $S$-matrix calculations (SM) of Rayleigh scattering amplitudes adequately treat elastic photon scattering by bound electrons even at energies close to the binding energy. Of the simpler approximations, the RFF + CCL and MRFF + CCL predictions using the relativistic form factor $f(q)$ and modified relativistic form factor $g(q)$ plus angle-independent high-energy-limit-corrected anomalous scattering factors $f_{C C L}^{\prime}$ and $g_{C C L}^{\prime}$ (without the Jensen term) are found not to be distinguished from the SM predictions by experiment. Theoretical predictions using anomalous scattering factors without a correct high-energy limit (RFF $+\mathrm{CL}$ ) are found to be clearly inferior to high-energy-limit-corrected predictions $(\mathrm{RFF}+\mathrm{CCL}$ and MRFF + CCL). Form-factor predictions without anomalous scattering factors (MRFF, NFF, and RFF) are found to be still worse.

The accuracy of the experiment has not yet fully reached that of experiments using radioactive photon sources. This may be seen, e.g., from a comparison of the present work with the scattering experiment on $\mathrm{Kr}$ and $\mathrm{Xe}$ at $59.5 \mathrm{keV}$ (Ref. 5). It seems worthwhile to discuss the reasons for this fact in order to deduce some recommendations for the improvement of the experimen- 
TABLE IX. Comparison of experiment and theory for scattering of parallel-polarized photons by xenon; SM is the $S$ matrix theory; RFF is the relativistic form factor; MRFF is the modified relativistic form factor; CCL is the angle-independent, high-energy-limit-corrected, anomalous scattering factors. The small contribution due to the nuclear Thomson amplitude is included. Numbers in square brackets are the associated power of 10 .

\begin{tabular}{|c|c|c|c|c|c|}
\hline \multirow[b]{2}{*}{$E(\mathrm{keV})$} & \multirow[b]{2}{*}{$\Theta(\operatorname{deg})$} & \multicolumn{4}{|c|}{$d \sigma_{\|} d \Omega\left(r_{0}^{2} / \mathrm{sr}\right)$} \\
\hline & & Experiment & SM & $\mathrm{RFF}+\mathrm{CCL}$ & $\mathrm{MRFF}+\mathrm{CCL}$ \\
\hline \multirow[t]{3}{*}{21.2} & 60 & $1.22 \pm 0.40[2]$ & $8.87[1]$ & $9.12[1]$ & $9.14[1]$ \\
\hline & 90 & & $1.79[-2]$ & 0 & 0 \\
\hline & 120 & $4.5 \pm 1.1[1]$ & $3.69[1]$ & $3.64[1]$ & $3.66[1]$ \\
\hline \multirow[t]{3}{*}{28.5} & 60 & $4.9 \pm 1.8[1]$ & $5.12[1]$ & $5.28[1]$ & $5.31[1]$ \\
\hline & 90 & & $1.21[-2]$ & 0 & 0 \\
\hline & 120 & $1.8 \pm 0.6[1]$ & $1.34[1]$ & $1.32[1]$ & $1.35[1]$ \\
\hline \multirow[t]{3}{*}{31.2} & 60 & $3.9 \pm 1.0[1]$ & $4.11[1]$ & $4.21[1]$ & $4.24[1]$ \\
\hline & 90 & & $9.72[-3]$ & 0 & 0 \\
\hline & 120 & $8 \pm 1$ & 8.64 & 8.28 & 8.56 \\
\hline \multirow[t]{3}{*}{33.95} & 60 & & $2.68[1]$ & $2.74[1]$ & $2.76[1]$ \\
\hline & 90 & & $5.01[-3]$ & 0 & 0 \\
\hline & 120 & & 3.25 & 3.12 & 3.30 \\
\hline \multirow[t]{3}{*}{34.26} & 60 & & $2.29[1]$ & $2.36[1]$ & $2.38[1]$ \\
\hline & 90 & & $3.65[-3]$ & 0 & 0 \\
\hline & 120 & & 2.03 & 2.04 & 2.16 \\
\hline \multirow[t]{3}{*}{34.42} & 60 & & $2.05[1]$ & $2.01[1]$ & $1.99[1]$ \\
\hline & 90 & & $2.28[-3]$ & 0 & 0 \\
\hline & 120 & & 1.28 & 1.16 & 1.15 \\
\hline \multirow[t]{3}{*}{35.36} & 60 & $4.0 \pm 1.9[1]$ & $2.91[1]$ & $2.95[1]$ & $2.97[1]$ \\
\hline & 90 & & $9.32[-3]$ & 0 & 0 \\
\hline & 120 & $8 \pm 6$ & 6.17 & 6.11 & 6.20 \\
\hline \multirow[t]{3}{*}{39.8} & 60 & $3.6 \pm 2.4[1]$ & $2.95[1]$ & $3.01[1]$ & $3.03[1]$ \\
\hline & 90 & & $1.31[-2]$ & 0 & 0 \\
\hline & 120 & $1.2 \pm 0.5[1]$ & 7.67 & 7.39 & 7.61 \\
\hline \multirow[t]{3}{*}{43.7} & 60 & $3.0 \pm 0.9[1]$ & $2.46[1]$ & $2.51[1]$ & $2.55[1]$ \\
\hline & 90 & & $1.61[-2]$ & 0 & 0 \\
\hline & 120 & $9 \pm 4$ & 7.15 & 6.88 & 7.10 \\
\hline
\end{tabular}

tal technique.

Apart from small absorption effects, radioactive sources emit photons isotropically and with an intensity depending in a well-known way on time. For two reasons, these two properties are essential for the high accuracy of cross sections measured with radioactive sources: (i) Rather large scatterers and solid angles can be used, leading to a good statistical accuracy of experimental data. All effects of geometry and absorption are well accounted for via an appropriate geometrical factor. (ii) The efficiency of the photon detector is eliminated from the evaluation of cross sections by measuring the source strength with the same detector, placed at a large distance from the source. Then, the intensity of the incident photons for the small distance used in the scattering experiment is obtained by only using the inversesquare law for the distance dependence of intensity and the well-known absorption coefficient for photons in air (taken, e.g., from Ref. 21). As a consequence, measurements of relative photon intensities yield absolute differential scattering cross sections with high precision.

Synchrotron radiation, on the other hand, is emitted in the bending magnets (or, sometimes, the wigglers) of an electron storage ring, essentially into the direction of the tangent to the electron orbit. Both the intensity and the polarization vary rapidly across a narrow angular interval around that direction (see, e.g., Ref. 23). Any changes of the position, the diameter, or the divergence of the orbiting electron beam lead to considerable variations of the intensity and the polarization of the collimated SR beam used for the experiment.

Most of the existing large electron storage rings (among them DORIS II) were designed for experiments in high-energy physics. They have, at some interaction points in the straight sections of the ring, a fixed position as well as a small cross section of the electron beam. As a consequence, the properties of the electron beam in the bending magnets are far from being optimized with respect to the needs of SR users. Especially during high-energy runs with parasitic use of SR, the position 
and the shape of the electron beam in the bending magnets may depend strongly and often irregularly on time, due to efforts to stabilize the beam at the interaction points.

The intensity of a source of SR cannot be reduced by a large, but precisely known factor. This fact makes the calibration of the photon detector quite difficult in comparison to an experiment using a radioactive photon source. At present, the only practical method for calibrating the detector is attenuating the direct beam by a thick absorber. However, as explained in Sec. III, the presence of harmonics in the monochromatized beam may heavily affect the calibration procedure.

In order to achieve a precision equal to, or even better than, that of experiments using radioactive photon sources, experiments using SR should be improved in several ways. (i) First of all, a dedicated storage ring, with beam optics optimized for the utilization of SR, is an essential prerequisite. It would provide a photon beam having better stability and, possibly, a larger degree of linear polarization. Only if $P$ is close to 1 can the scattering cross section $d \sigma_{\|} / d \Omega$ be measured with good accuracy. (ii) Monochromators which do not transmit harmonics should be used. (iii) The attenuation coefficient of the absorber used in the calibration of the detector should be known more precisely than at present. With these and, perhaps, additional measures, photon scattering experiments using SR certainly will become an excellent tool for a systematic and comprehensive investigation of Rayleigh scattering in the hard-x-ray regime.

\section{ACKNOWLEDGMENTS}

The authors wish to thank Professor Dr. U. Bonse and Professor Dr. K. Fischer for making available the two-axis diffractometer for the experiment, and $\mathbf{H}$. Schenk-Strauss for his help during part of the measurements. They are grateful to Dr. D. T. Cromer for providing a copy of the computer program FPRIME. This work was supported by the Bundesministerium für Forschung und Technologie under Contract No. PS05273-SD, and by the U.S. Department of Energy under Contract No. DE-AC04-76DP00789.
*Present address: IBM Deutschland G.m.b.H., D-7032 Sindelfingen, Federal Republic of Germany.

†Present address: Philips G.m.b.H., D-2300 Kiel, Federal Republic of Germany.

†Present address: Deutsches Elektronen-Synchrotron DESY, D-2000 Hamburg 52, Federal Republic of Germany.

${ }^{1}$ M. Gavrila, in $X$-Ray and Atomic Inner-Shell Physics (Oregon, 1982), Proceedings of the Conference on X-ray and Atomic Inner-Shell Physics, AIP Conf. Proc. No. 94, edited by B. Crasemann (AIP, New York, 1982), p. 357.

${ }^{2}$ L. Kissel and R. H. Pratt, in Atomic Inner-Shell Physics, edited by B. Crasemann (Plenum, New York, 1985).

${ }^{3}$ P. P. Kane, L. Kissel, R. H. Pratt, and S. C. Roy, Phys. Rep. 140, 75 (1986).

${ }^{4}$ M. Schumacher and A. Stoffregen, Z. Phys. A 283, 15 (1977).

${ }^{5}$ F. Smend and H. Czerwinski, Z. Phys. D 1, 139 (1986).

${ }^{6}$ W. Mückenheim and M. Schumacher, J. Phys. G 6, 1237 (1980).

${ }^{7}$ M. Schumacher, F. Smend, P. Rullhusen, W. Mückenheim, and H. G. Börner, Inst. of Phys. Conf. Ser. 62, 598 (1982).

${ }^{8}$ K. G. Tirsell, V. W. Slivinsky, and P. J. Ebert, Phys. Rev. A 12, 2426 (1975).

${ }^{9}$ L. Kissel, R. H. Pratt, and M. Gavrila (unpublished).

${ }^{10}$ J. H. Hubbell, W. J. Veigele, E. A. Briggs, R. T. Brown, D. T. Cromer, and R. J. Howerton, J. Phys. Chem. Ref. Data 4, 471 (1975); 6, 615(E) (1977).

${ }^{11}$ J. H. Hubbell and I. Øverbø, J. Phys. Chem. Ref. Data 8, 69
(1979).

${ }^{12}$ W. Franz, Z. Phys. 98, 314 (1936).

${ }^{13}$ D. Schaupp, M. Schumacher, F. Smend, P. Rullhusen, and J. H. Hubbell, J. Phys. Chem. Ref. Data 12, 467 (1983).

14J. J. Sakurai, Advanced Quantum Mechanics (AddisonWesley, Reading, Mass., 1967).

${ }^{15}$ D. T. Cromer and D. J. Liberman, J. Chem. Phys. 53, 1891 (1970).

${ }^{16}$ D. T. Cromer, J. Appl. Crystallog. 16, 437 (1983).

${ }^{17}$ D. Y. Smith, Phys. Rev. A 35, 3381 (1987).

${ }^{18}$ M. S. Jensen, Phys. Lett. 74A, 47 (1979); see also M. S. Jensen, J. Phys. B 13, 4337 (1980).

${ }^{19}$ U. Bonse, Th. Risse, U. Dretzler, S. Krasnicki, W. Schildkamp, H. Schenk-Strauss, K. H. Ehses, and K. Fischer, Z. Kristallogr. 162, 31 (1983).

${ }^{20}$ F. Smend, D. Schaupp, H. Czerwinski, A. H. Millhouse, and H. Schenk-Strauss, Nucl. Instrum. Methods A 241, 290 (1985).

${ }^{21}$ E. F. Plechaty and J. R. Terrall, University of California Radiation Laboratory, Report No. 50400, 1968 (unpublished).

${ }^{22}$ H. Czerwinski, F. Smend, D. Schaupp, M. Schumacher, A. H. Millhouse, and H. Schenk-Strauss, Z. Phys. A 322, 183 (1985).

${ }^{23}$ Synchrotron Radiation, Techniques and Applications, Vol. 10 of Topics in Current Physics, edited by C. Kunz (Springer, Berlin, 1979). 